\title{
Exact distributions of the number of distinct and common sites visited by $N$ independent random walkers
}

\author{
Anupam Kundu, ${ }^{1}$ Satya N. Majumdar, ${ }^{1}$ and Grégory Schehr ${ }^{1}$ \\ ${ }^{1}$ Laboratoire de Physique Théorique et Modèles Statistiques (UMR 8626 du CNRS), \\ Université Paris-Sud, Bât. 100, 91405 Orsay Cedex, France
}

\begin{abstract}
We study the number of distinct sites $S_{N}(t)$ and common sites $W_{N}(t)$ visited by $N$ independent one dimensional random walkers, all starting at the origin, after $t$ time steps. We show that these two random variables can be mapped onto extreme value quantities associated to $N$ independent random walkers. Using this mapping, we compute exactly their probability distributions $P_{N}^{d}(S, t)$ and $P_{N}^{d}(W, t)$ for any value of $N$ in the limit of large time $t$, where the random walkers can be described by Brownian motions. In the large $N$ limit one finds that $S_{N}(t) / \sqrt{t} \propto 2 \sqrt{\log N}+\widetilde{s} /(2 \sqrt{\log N})$ and $W_{N}(t) / \sqrt{t} \propto \widetilde{w} / N$ where $\widetilde{s}$ and $\widetilde{w}$ are random variables whose probability density functions (pdfs) are computed exactly and are found to be non trivial. We verify our results through direct numerical simulations.
\end{abstract}

PACS numbers: 05.40.-a, 02.50.-r, 05.40.Jc

In elementary set theory, two fundamental concepts are the union and the intersection of a number of $N$ sets. While the union consists of all distinct elements of the collection of sets, the intersection consists of common elements of all the sets. These two notions appear naturally in everyday life: for example the area of common knowledge or the whole range of different interests amongst the members of a society would define respectively its stability and activity. In an habitat of $N$ animals, the union of the territories covered by different animals sets the geographical range of the habitat, while the intersection refers to the common area (e. g. a water body) frequented by all animals.

In statistical physics, these two objects are modeled respectively by the number of distinct and common sites visited by $N$ random walkers (RWs). The knowledge about the number of distinct sites has applications ranging from the annealing of defects in crystals [1, 2] and relaxation processes [3 [ $[$ ] to the spread of populations in ecology [7, 8] or to the dynamics of web annotation systems [9]. Similarly the knowledge about the common area frequented by endangered animals is very useful for their daily health caring. Likewise, in the energy transport through a series of independent disordered samples, the energy output will depend on the number of energy levels common to all these materials.

Dvoretzky and Erdös [10] first studied the average number of distinct sites $\left\langle S_{1}(t)\right\rangle$ visited by a single $t$ step RW in $d$-dimensions, subsequently studied in [1113]. Larralde et al. generalized this to $N$ independent, $t$-step walkers moving on a $d$-dimensional lattice [14]. They found three regimes of growth (early, intermediate and late) for the average number of distinct sites $\left\langle S_{N}(t)\right\rangle$ as a function of time. These three regimes are separated by two $N$-dependent times scales [14]. In particular they showed that in $d=1$ and $t \gg \sqrt{\log N}$, $\left\langle S_{N}(t)\right\rangle \propto \sqrt{4 D t \log N}$ where $D$ is the diffusion constant of a single walker. Recently Majumdar and Tamm [15] studied the complementary quantity, namely the number of common sites $W_{N}(t)$ visited by $N$ walkers, each of $t$ steps, and found analytically a rich asymptotic late time growth of the average $\left\langle W_{N}(t)\right\rangle$. They showed that in the $(N-d)$ plane there are three distinct phases separated by two critical lines $d=2$ and $d_{c}(N)=2 N /(N-1)$, with $\left\langle W_{N}(t)\right\rangle \sim t^{\nu}$ at late times where the growth exponent $\nu=d / 2$ (for $d<2), \nu=N-d(N-1) / 2$ [for $2<d<d_{c}(N)$ ] and $\nu=0$ [for $d>d_{c}(N)$ ] (see also [16]). In particular, in $d=1,\left\langle W_{N}(t)\right\rangle \sim \sqrt{4 D t}$ where the prefactor depends on $N$. However, most of these studies were limited to the average number of distinct or common sites, and there exists virtually no information about their full probability distributions, e.g. the probabilities $P_{N}^{d}(S, t)$ that $S_{N}(t)=S$ and $P_{N}^{c}(W, t)$ that $W_{N}(t)=W$.

Computing these distributions for general $d$ dimensional space is highly non trivial. Indeed, although the $N$ walkers are independent, conditioning their trajectories to a given number of distinct (or common) visited sites introduces strong effective correlations between them. In $d=1$, we show here that these random variables $S_{N}(t)$ and $W_{N}(t)$ can be mapped onto extreme values (nearest and furthest displacements) associated to $N$ independent walkers. This connection to extreme value statistics (EVS) allows us to compute $P_{N}^{d}(S, t)$ and $P_{N}^{c}(W, t)$ exactly for $t$ large and arbitrary $N$. We show that the induced correlations between the walkers persist even for $N \rightarrow \infty$ where the limiting distributions are not given by EVS of independent random variables, as erroneously argued in the previous study of $S_{N}(t)$ [14].

We consider $N$ independent and identical $t$-step RWs $x_{1}(\tau), x_{2}(\tau), \cdots, x_{N}(\tau)$ on a 1-d lattice, all starting at the origin. For convenience, we set the diffusion constant of the walkers $D=\frac{1}{2}$. Distinct sites are those that are visited at least once by at least one of the $N$ walkers [14], while common sites correspond to sites visited individually at least once by all the $N$ walkers [15]. We denote by $M_{i}$ and $m_{i}$ respectively the maximum and the min- 


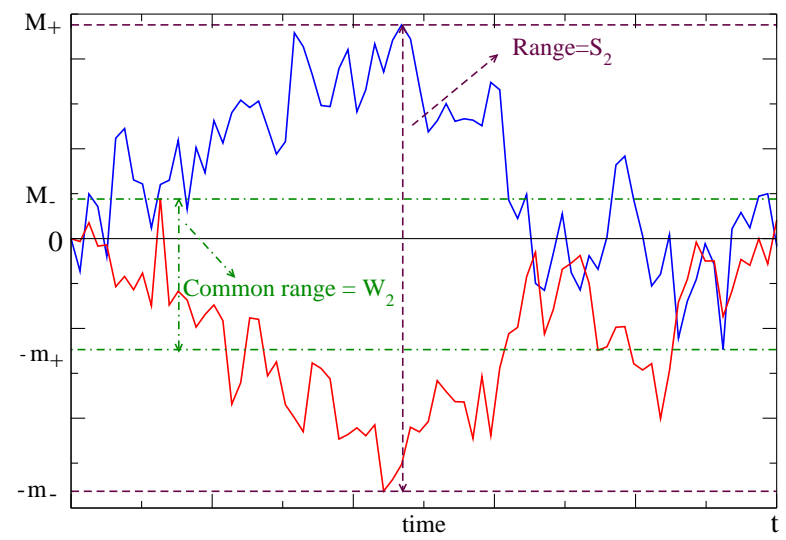

FIG. 1. (Color Online) Schematic diagram of 2 independent RWs, where $M_{+}, M_{-}, m_{+}, m_{-}$and $S_{2}, W_{2}$ are shown (1) 2).

imum displacements of the $i^{\text {th }}$ walker $x_{i}$ up to time $t$. The number of distinct sites visited, $S_{N}$ [17], is then the sum of the range on the positive (+ve) side, $M_{+}$, and the range on the negative (-ve) side $m_{-}$(see Fig. 1):

$S_{N}=M_{+}+m_{-}, M_{+}=\max _{1 \leq i \leq N} M_{i}, m_{-}=-\min _{1 \leq i \leq N} m_{i}$

Similarly, the number of common sites visited, $W_{N}$, is the common span on the +ve axis plus the common span $m_{+}$on the -ve axis:

$W_{N}=M_{-}+m_{+}, M_{-}=\min _{1 \leq i \leq N} M_{i}, m_{+}=-\max _{1 \leq i \leq N} m_{i}$

Eqs. (1) and (2) establish a precise connection between $S_{N}$ and $W_{N}$ and the EVS of $N$ independent RW's.

In the limit of large $t$, the lattice RWs converge to Brownian motions (BMs). Hence for large $t$, the probability distributions $P_{N}^{d}(S, t)$ and $P_{N}^{c}(W, t)$ take the scaling form

$P_{N}^{d}(S, t)=\frac{1}{\sqrt{2 t}} p_{N}^{d}\left(\frac{S}{\sqrt{2 t}}\right), P_{N}^{d}(W, t)=\frac{1}{\sqrt{2 t}} p_{N}^{d}\left(\frac{W}{\sqrt{2 t}}\right)$

where $p_{N}^{d}(s)$ is the probability density function (pdf) of the span or range, $s=S / \sqrt{2 t}$, and $p_{N}^{c}(w)$ is the pdf of the common span or common range, $w=W / \sqrt{2 t}$, for $N$ independent BMs (see Fig. 1) on the unit time interval [18]. The rescaled quantities $S_{N} / \sqrt{2 t}$ and $W_{N} / \sqrt{2 t}$ in (3) are given by (11) and (2) where $M_{ \pm}, m_{ \pm}$are replaced by their counterparts $\widetilde{M}_{ \pm}=M_{ \pm} / \sqrt{2 t}$ and $\widetilde{m}_{ \pm}=m_{ \pm} / \sqrt{2 t}$ corresponding to $N$ independent BMs on the unit time interval.

It is useful to summarize our main results. We obtain exactly, for any $N$, the $\operatorname{pdfs} p_{N}^{d}(s)$ and $p_{N}^{c}(w)$ as presented in (12) and (15) along with (8) and (9). The moments can also be computed explicitly [19]. The tails of the pdfs can be derived explicitly:

$$
p_{N}^{d}(s) \sim\left\{\begin{array}{l}
a_{N} s^{-5} \exp \left[-N \pi^{2} /\left(4 s^{2}\right)\right], s \rightarrow 0, \\
b_{N} \exp \left(-s^{2} / 2\right), s \rightarrow \infty
\end{array}\right.
$$

and

$$
p_{N}^{c}(w) \sim\left\{\begin{array}{l}
c_{N} w, w \rightarrow 0 \\
d_{N} w^{1-N} \exp \left(-N w^{2}\right), w \rightarrow \infty,
\end{array}\right.
$$

where $a_{N}, b_{N}, c_{N}$ and $d_{N}$ are computable constants (see below). For $N \rightarrow \infty$, one finds that both pdfs approach a non trivial limiting form

$$
\begin{aligned}
& p_{N}^{d}(s) \sim 2 \sqrt{\log N} \mathcal{D}(2 \sqrt{\log N}(s-2 \sqrt{\log N})), \\
& \mathcal{D}(\widetilde{s})=2 e^{-\widetilde{s}} K_{0}\left(2 e^{-\widetilde{s} / 2}\right)
\end{aligned}
$$

where $K_{n}(x)$ denote the modified Bessel functions, and

$$
p_{N}^{c}(w)=N \mathcal{C}(N w), \mathcal{C}(\widetilde{w})=\frac{4}{\pi} \widetilde{w} e^{-\frac{2}{\sqrt{\pi}} \widetilde{w}}, \widetilde{w}>0 .
$$

Note that $\mathcal{D}(\tilde{s})$ (6) is not the Gumbel distribution, as it was initially argued in [14]. Remarkably the same distribution $\mathcal{D}(\tilde{s})$ also appears as the limiting distribution of the maximum of a large collection of logarithmically correlated random variables on a circle [20]. We check indeed $\int_{-\infty}^{\tilde{s}} \mathcal{D}\left(\tilde{s}^{\prime}\right) d \tilde{s}^{\prime}=2 e^{-\tilde{s} / 2} K_{1}\left(2 e^{-\tilde{s} / 2}\right)$, as obtained in [20]. Incidentally, logarithmically correlated random variables have been the subject of several recent studies 20 22 because they exhibit freezing phenomena, akin to the replica symmetry breaking scenario found in mean field spin glass models [23]. As a byproduct of our computation, we show that $\mathcal{D}(\tilde{s})$ is the convolution of two independent Gumbel distributions.

We start by computing the joint cumulative distribution functions (jcdf) $\mathbf{P}_{d}\left(l_{1}, l_{2}\right)$ $=\operatorname{Pr} .\left(\widetilde{M}_{+} \leq l_{1}, \widetilde{m}_{-} \leq l_{2}\right)$, relevant for $p_{N}^{d}(s)$ and the jcdf $\mathbf{P}_{c}\left(j_{1}, j_{2}\right)=\operatorname{Pr} .\left(\widetilde{M}_{-} \geq j_{1}, \widetilde{m}_{+} \geq j_{2}\right)$ relevant for $p_{N}^{c}(w)$. Since all the $N$ BMs are identical and independent, $\mathbf{P}_{d}\left(l_{1}, l_{2}\right)=g^{N}\left(l_{1}, l_{2}\right)$, where $g\left(l_{1}, l_{2}\right)=\operatorname{Pr} .\left(\widetilde{M} \leq l_{1}, \widetilde{m} \geq-l_{2}\right)$ is the jcdf of the maximum $\widetilde{M}$ and the minimum $\widetilde{m}$ for a single $\mathrm{BM}$ on the unit time interval. It can be computed by the standard method of images [24]:

$g\left(l_{1}, l_{2}\right)=\frac{2}{\pi} \sum_{n=0}^{\infty} \frac{1}{n+\frac{1}{2}} \sin \left(\frac{(2 n+1) \pi l_{2}}{l_{1}+l_{2}}\right) e^{-\left(\frac{\left(n+\frac{1}{2}\right) \pi}{l_{1}+l_{2}}\right)^{2}}$

Similarly, $\mathbf{P}_{c}\left(j_{1}, j_{2}\right)=h^{N}\left(j_{1}, j_{2}\right)$ where $h\left(j_{1}, j_{2}\right)=$ $\operatorname{Pr} .\left(\widetilde{M} \geq j_{1}, \widetilde{m} \leq-j_{2}\right)$ reads:

$$
h\left(j_{1}, j_{2}\right)=1-\operatorname{erf}\left(j_{1}\right)-\operatorname{erf}\left(j_{2}\right)+g\left(j_{1}, j_{2}\right),
$$

where $\operatorname{erf}(x)=(2 / \sqrt{\pi}) \int_{0}^{x} e^{-y^{2}} d y, \operatorname{erf}\left(j_{1}\right)=\operatorname{Pr} \cdot(\widetilde{M} \leq$ $\left.j_{1}\right)$ and $\operatorname{erf}\left(j_{2}\right)=\operatorname{Prob}\left(\widetilde{m} \geq-j_{2}\right)$. From the joint pdf 
$\frac{\partial^{2} \mathbf{P}_{d}\left(l_{1}, l_{2}\right)}{\partial l_{1} \partial l_{2}}$ and using (1), we obtain

$$
p_{N}^{d}(s)=\int_{0}^{\infty} d l_{1} \int_{0}^{\infty} d l_{2} \delta\left(s-l_{1}-l_{2}\right) \frac{\partial^{2} g^{N}}{\partial l_{1} \partial l_{2}}
$$

with $g \equiv g\left(l_{1}, l_{2}\right)$. Similarly, from the joint pdf $\frac{\partial^{2} \mathbf{P}_{c}\left(j_{1}, j_{2}\right)}{\partial j_{1} \partial j_{2}}$ and using (2) we obtain,

$$
p_{N}^{c}(w)=\int_{0}^{\infty} d j_{1} \int_{0}^{\infty} d j_{2} \delta\left(w-j_{1}-j_{2}\right) \frac{\partial^{2} h^{N}}{\partial j_{1} \partial j_{2}},
$$

with $h \equiv h\left(j_{1}, j_{2}\right)$. For small values of $N$, the double integrals in (10) and (11) can be performed explicitly and numerical simulations confirm these exact results [19] . Below we provide a physical interpretation of these formulas (10, 11) and perform, separately, their asymptotic analysis both for small and large arguments. We also analyze their limiting form for $N \rightarrow \infty$.

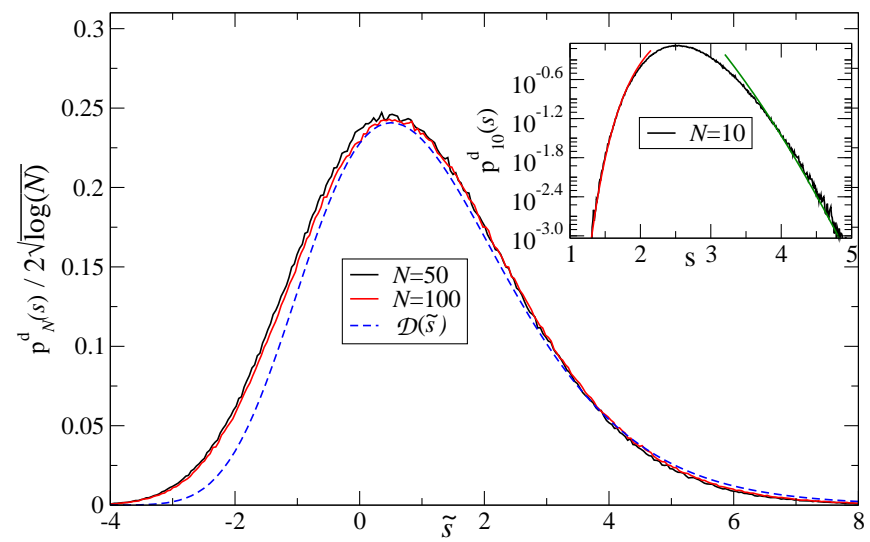

FIG. 2. (Color online) Plot of $p_{N}^{d}(s) /(2 \sqrt{\log N})$ as a function of $\widetilde{s}=2 \sqrt{\log N}(s-2 \sqrt{\log N})$. The dotted line indicates the exact asymptotic results for $N \rightarrow \infty, \mathcal{D}(\widetilde{s})$ in (6). Inset: Plot of $p_{10}^{d}(s)$, obtained from simulation, compared with its asymptotic behavior (4).

Distinct sites : To find the tails of $p_{N}^{d}(s)$ at small and large $s$ for finite $N$, we rewrite (10) as

$$
\begin{aligned}
& p_{N}^{d}(s)=\int_{0}^{s} d l_{2} \Psi_{d}\left(s-l_{2}, l_{2}\right) \text { where } \\
& \Psi_{d}\left(l_{1}, l_{2}\right)=N g^{N-1} \frac{\partial^{2} g}{\partial l_{1} \partial l_{2}}+N(N-1) g^{N-2} \frac{\partial g}{\partial l_{1}} \frac{\partial g}{\partial l_{2}} .
\end{aligned}
$$

We interpret the two contributions in $\Psi_{d}\left(l_{1}, l_{2}\right)$ as follows [19]: the first term corresponds to a configuration where one particle explores a region $\left[-l_{2}, s-l_{2}\right]$ (we call it a box) of size $s$ in unit time interval, such that its maximum is at $s-l_{2}$ and minimum is at $-l_{2}$, while all the other $(N-1)$ particles stay inside this box. On the other hand, the second term corresponds to a configuration where two particles create, in a different way, the same box $\left[-l_{2}, s-l_{2}\right]$ of size $s$ : one of the two particles has its maximum at $s-l_{2}$ and minimum larger than $-l_{2}$ while the second particle has its minimum at $-l_{2}$ and maximum below $s-l_{2}$ and all other $(N-2)$ particles stay strictly inside this box.

When $s \rightarrow 0$ in (12), one can replace $g\left(l_{1}, l_{2}\right)$ (8) by its asymptotic behavior when $l_{1}, l_{2} \rightarrow 0$ where $g\left(l_{1}, l_{2}\right) \sim \frac{4}{\pi} \sin \left(\frac{\pi l_{2}}{l_{1}+l_{2}}\right) e^{-\frac{\pi^{2}}{4\left(l_{1}+l_{2}\right)^{2}}}$. Inserting it in (12), we see that both terms in (12) contribute equally. After integration over $l_{2}$, one then obtains the result announced in (4) for $s \rightarrow 0$ with $a_{N}=4 \pi^{3 / 2} N(N-1)\left(\frac{4}{\pi}\right)^{N-2} \frac{\Gamma\left(\frac{N-1}{2}\right)}{\Gamma\left(\frac{N}{2}\right)}$, where $\Gamma(x)$ is the Gamma function. To perform the large $s$ asymptotic of $p_{N}^{d}(s)$ we use the Poisson summation formula: $g\left(l_{1}, l_{2}\right)=$ $\sum_{m=0}^{\infty}(-1)^{m}\left[\operatorname{erf}\left[m\left(l_{1}+l_{2}\right)+l_{1}\right]+\operatorname{erf}\left[m\left(l_{1}+l_{2}\right)+l_{2}\right]\right]$. We use this form to evaluate the integrand in (12) in the limit $s \rightarrow \infty$. We see that the first term in (12), which corresponds to create a box $\left[-l_{2}, s-l_{2}\right]$ with one particle, decreases as $e^{-\left(s+l_{2}\right)^{2}} e^{-l_{2}^{2}}$ whereas the second term where the same box is created by two particles decreases as $e^{-\left(s-l_{2}\right)^{2}} e^{-l_{2}^{2}}$. Since $l_{2}$ is always $+\mathrm{ve}$, the two particles term wins over the one particle term when $s \rightarrow \infty$ : this is physically understandable because creating a very large span with two particles is more likely than creating the same one with a single particle. It also follows from this analysis that the integral over $l_{2}$ in (12) is dominated by $l_{2} \sim \mathcal{O}(s)$, which yields finally the large $s$ behavior announced in (4) with $b_{N}=2 N(N-1) / \sqrt{\pi}$. In Fig. 2 we verify that the small and large $s$ asymptotics of $p_{N}^{d}(s)$ given in (4), for $N=10$, describe very well, without any fitting parameter, the distribution obtained from direct simulation, without any fitting parameter.

What happens for large $N$ ? The typical scale of the fluctuations of $S_{N} / \sqrt{2 t}$ can be estimated from the relations with EVS (11). The variables $\widetilde{M}_{i}^{\prime}$ 's, with $i=$ $1, \cdots, N$, which are the maxima of the $i^{\text {th }} \mathrm{BM}$ on the unit interval, are i.i.d. variables. Their common pdf is known to be a half-Gaussian, $p(M)=(2 / \sqrt{\pi}) e^{-M^{2}}, M>0$. The same holds for the variables $-\widetilde{m}_{i}$ 's. Hence, for large $N$, standard results of EVS [25] state that the typical value of $\widetilde{M}_{+}=\max _{1 \leq i \leq N} \widetilde{M}_{i}$ is $\mathcal{O}(\sqrt{\log N})$ while its fluctuations are of order $1 / \sqrt{\log N}$ and governed by a Gumbel distribution. The same also holds for $\widetilde{m}_{-}=-\min _{1 \leq i \leq N} \widetilde{m}_{i}$. For large $N$, these two extremes become uncorrelated as the global maximum and global minimum are most likely reached by two independent walkers. Hence one gets

$$
g^{N}\left[\mu_{N}+\frac{\tilde{l}_{1}}{2 \mu_{N}}, \mu_{N}+\frac{\tilde{l}_{2}}{2 \mu_{N}}\right] \underset{N \rightarrow+\infty}{\longrightarrow} e^{-e^{-\tilde{l}_{1}}} e^{-e^{-\tilde{l}_{2}}}
$$

with $\mu_{N}=\sqrt{\log N}$. Inserting (13) in (10) with $\widetilde{s}=$ $2 \mu_{N}\left(s-2 \mu_{N}\right)$ one finds

$$
p_{N}^{d}(s) \sim 2 \sqrt{\log N} \int_{-\infty}^{\infty} d \widetilde{l}_{2} e^{-\widetilde{s}} e^{-e^{-\widetilde{l}_{2}}} e^{-e^{-\left(\tilde{s}-\tilde{l}_{2}\right)}},
$$

which can be evaluated explicitly to give (6). In Fig. 2 we $\operatorname{plot} p_{N}^{d}(s) / 2 \sqrt{\log N}$ against $\widetilde{s}$ for $N=50$ and 100. They 
show a relatively good agreement with the exact result $\mathcal{D}(\tilde{s})$ after an overall shift of order $\mathcal{O}(1 / \log N)$ along the $x$-axis, thus revealing, as expected, a slow convergence towards the asymptotic result. In [14] the authors argued that the limiting distribution should be a Gumbel distribution, overlooking the fact that it is actually the convolution of two Gumbel distributions, as in (14). In particular, for large $\tilde{s}, \mathcal{D}(\tilde{s}) \sim \tilde{s} e^{-\tilde{s}}$, while the Gumbel distribution decays as a pure exponential.

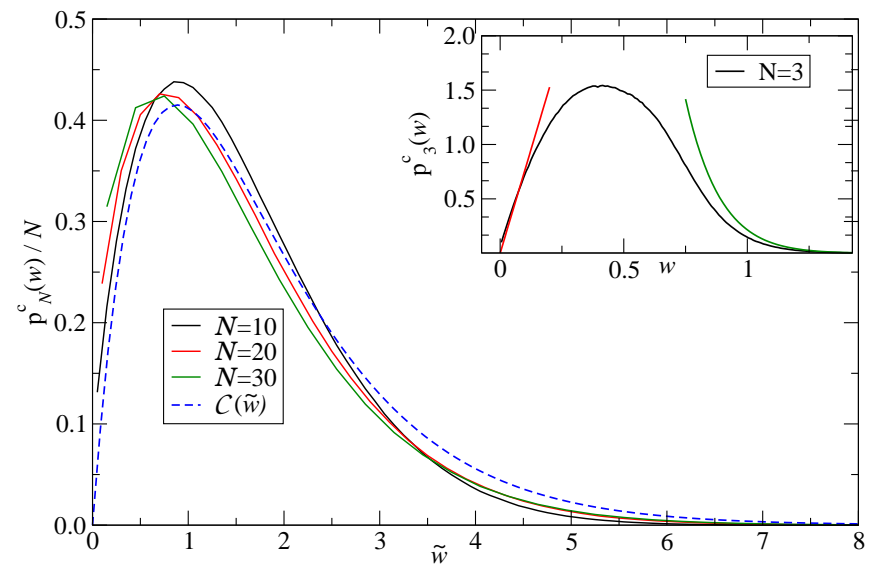

FIG. 3. (Color online) Plot of $p_{N}^{c}(w) / N$ as a function of $\widetilde{w}=$ $N w$. The dotted line indicates the exact asymptotic results for $N \rightarrow \infty, \mathcal{C}(\widetilde{w})$ in (7). Inset: Plot of $p_{3}^{d}(w)$, obtained from simulation, compared with its asymptotic behavior (5).

Common sites : To find the small and large $w$ asymptotics of $P_{N}^{c}(w)$ we write (11) as

$$
\begin{aligned}
& p_{N}^{c}(w)=\int_{0}^{w} d j_{2} \Psi_{c}\left(w-j_{2}, j_{2}\right) \text { where } \\
& \Psi_{c}\left(j_{1}, j_{2}\right)=N h^{N-1} \frac{\partial^{2} h}{\partial j_{1} \partial j_{2}}+N(N-1) h^{N-2} \frac{\partial h}{\partial j_{1}} \frac{\partial h}{\partial j_{2}} .
\end{aligned}
$$

In (15), one interprets the first term as one single particle creating a common span $\left[-j_{2}, w-j_{2}\right]$ of size $w$ and the second term as two particles collaboratively creating the same common span (in a unit time interval) [19]. In both cases, the remaining particles are such that their maxima are above $w-j_{2}$ and their minima are below $-j_{2}$. When $w \rightarrow 0$ in (15), $h\left(j_{1}, j_{2}\right)$ can be replaced by its asymptotic behavior for small $j_{1}, j_{2}: h\left(j_{1}, j_{2}\right) \sim$ $\left(1-\frac{2}{\sqrt{\pi}}\left(j_{1}+j_{2}\right)\right)$. Integrating then over $j_{2}$ in (15) yields the small $w$ behavior in (5) with $c_{N}=4 N(N-1) / \pi$. Note that for very small $w$, it is much more likely to create a box of size smaller than $w$ with two particles (which occurs with a probability $\propto w^{2}$ ) than with a single one [which occurs with probability $\left.\propto \exp \left(-\pi^{2} / 4 w^{2}\right)\right]$. The former configurations thus dominate for small $w$.

To get the large $w$ behavior of $p_{N}^{c}(w)$, we estimate $h\left(j_{1}, j_{2}\right)$ for large $j_{1}$ (15). This is conveniently done by using the Poisson formula, which yields $h\left(j_{1}, j_{2}\right) \sim$ $\operatorname{erfc}\left(2 j_{1}+j_{2}\right)+\operatorname{erfc}\left(j_{1}+2 j_{2}\right)$. This estimate shows that for $w \gg \sqrt{\log N}$, the second term in (15) becomes subdominant compared to the first one. Hence for very large $w$ the leading contribution comes from the first term where we replace $h^{(N-1)}\left(w-j_{2}, j_{2}\right) \sim\left[\operatorname{erfc}\left(w+j_{2}\right)+\right.$ $\left.\operatorname{erfc}\left(2 w-j_{2}\right)\right]^{N-1}$ by $\operatorname{erfc}^{(N-1)}(w)$ as one can show that the integral over $j_{2}$ in (15) is dominated by the vicinity of $j_{2}=0$ [19]. This leads to the large $w$ behavior in (5) with $d_{N}=8 N / \pi^{N / 2}$. The asymptotic behaviors of $p_{N}^{c}(w)$ (5) have been verified numerically for $N=3$ in Fig. 3.

To obtain the typical scale of $W_{N} / \sqrt{2 t}$ for large $N$, we use its relation to EVS (2). From standard EVS for i.i.d. random variables [25], we know that $\widetilde{M}_{-}=$ $\min _{1 \leq i \leq N} M_{i}$, where $M_{i} \geq 0$ and distributed according to a half-Gaussian, is of order $\mathcal{O}\left(N^{-1}\right)$. Its pdf is given by a Weibull law, which is here an exponential distribution [25]. Indeed one has here $\operatorname{Pr} .\left(N \widetilde{M}_{-} \geq x\right)=e^{-\frac{2}{\sqrt{\pi}} x}$, $x>0$, as $N \rightarrow \infty$. The same holds for $\widetilde{m}_{+}$, which for large $N$ becomes independent of $\widetilde{M}_{-}$as both of them are reached by two independent walkers. Hence, from (2), $N W_{N} / \sqrt{2 t}$ is given by the convolution of two exponential laws:

$$
p_{N}^{c}(w) \sim N^{2}(4 / \pi) e^{-\frac{2}{\sqrt{\pi}} N w} \int_{0}^{w} d k \sim N \mathcal{C}(N w),
$$

with $\mathcal{C}(\widetilde{w})$ as announced in (7). We have also obtained this result [19] by a direct large $N$ expansion of (15). In Fig. 3 we plot $p_{N}^{c}(w) / N$ against $\widetilde{w}$ for $N=10,20$ and 30 and see that they both coincide with the function $\mathcal{C}(\tilde{w})$, although the convergence is rather slow.

Conclusion: We have achieved a complete analytic description of the pdfs of the number of distinct and common sites visited by $N$ independent RWs after $t$ time steps, for large $t$. We have also obtained interesting limiting distributions (6] (7) in the limit when $N \rightarrow \infty$. For distinct sites, we found an intriguing connection with the maximum of logarithmically correlated random variables on a circle [20].

One may wonder about the effects of interactions between the walkers. For instance, one can study nonintersecting (vicious) RWs [27]. An interesting situation is the case where all $N$ walkers start and end at the same point, while staying positive in the time interval $[0, t]$ (watermelons with a wall). In this case, the number of distinct sites $S_{N} / \sqrt{2 t}$ corresponds to the maximal height of these watermelons [28]. For large $N$, the pdf of $S_{N} / \sqrt{2 t} \propto \sqrt{N}$ properly shifted and scaled, converges to the Tracy-Widom distribution $\mathcal{F}_{1}$ [29], which describes the fluctuations of the largest eigenvalue of Gaussian orthogonal random matrices. On the other hand, the number of common sites $W_{N} / \sqrt{2 t}$ is related to the maximum of the lower path, the distribution of which is a very interesting open problem [31].

We thank A. Perret for a useful discussion. This research was supported by ANR grant 2011-BS04-01301 WALKMAT and in part by the Indo-French Centre 
for the Promotion of Advanced Research under Project $4604-3$.

[1] R. J. Beeler and A. J. Delaney, Phys. Rev. A 130, 962 (1963).

[2] R. J. Beeler, Phys. Rev. A 134, 1396 (1964).

[3] A. Blumen, J. Klafter and G. Zumofen, in Optical Spectroscopy of Glasses, edited by I. Zschokke (Reidel, New York, 1986), pp. 199-265.

[4] R. Czech, J. Chem. Phys. 91, 2498 (1989).

[5] P. Bordewijk, Chem. Phys. Lett. 32, 592 (1975).

[6] C. A. Condat, Phys. Rev. A 41, 3365 (1990).

[7] L. Edelstein-Keshet, Mathematical Models in Biology (Random House, New York, 1988).

[8] E. C. Pielou, An Introduction to Mathematical Ecology (Wiley-Interscience, New York, 1969);

[9] C. Cattuto, A. Barrat, A. Baldassari, G. Schehr and V. Loreto, Proc. Natl. Acad. Sci. USA 106, 10511 (2009).

[10] A. Dvoretzky and P. Erdös, in Proceedings of the Second Berkeley Symposium on Mathematical Statistics and Probability (University of California Press, Berkeley, 1951).

[11] G. H. Vineyard, J. Math. Phys. 4, 1191 (1963).

[12] E. W. Montroll and G. H. Weiss, J. Math. Phys. 6, 167 (1965).

[13] F. van Wijland and H. J. Hilhorst, J. Stat. Phys. 89, 119 (1997).

[14] H. Larralde, P. Trunfino, S. Havlin, H. E. Stanley, and
G. H. Weiss, Nature (London) 355, 423 (1992); Phys. Rev. A 45, 7128 (1992).

[15] S. N. Majumdar and M. V. Tamm, Phys. Rev. E 86, 021135 (2012).

[16] L. Turban, preprint arXiv: 1209.2527

[17] From now on, we drop the explicit $t$ dependence on $S_{N}, W_{N}, M_{i}, m_{i}$.

[18] with diffusion constant $D=1 / 4$.

[19] A. Kundu, S. N. Majumdar and G. Schehr, supplementary material.

[20] Y. V. Fyodorov and J.-Ph. Bouchaud, J. Phys. A: Math. Theor. 41, 372001 (2008).

[21] D. Carpentier and P. Le Doussal, Phys. Rev. E 63, 026110 (2001).

[22] Y. V. Fyodorov, P. Le Doussal and A. Rosso, J. Stat. Mech., P10005 (2009).

[23] M. Mézard, G. Parisi, M. Virasoro, Spin-Glass Theory and Beyond, (Singapore: World Scientific), (1997).

[24] S. Redner, A guide to first-passage processes, Cambridge University Press, Cambridge, 2001.

[25] E. J. Gumbel, Statistics of Extremes, Dover, (1958).

[26] K. L. Chung, Ark. Mat. 14(2), 155 (1976).

[27] M. E. Fisher, J. Stat. Phys. 34, 667 (1984).

[28] G. Schehr, S. N. Majumdar, A. Comtet and J. RandonFurling, Phys. Rev. Lett. 101, 150601 (2008).

[29] P. J. Forrester, S. N. Majumdar and G. Schehr, Nucl. Phys. B 844, 500 (2011); Erratum Nucl. Phys. B 857, 424 (2011).

[30] C. A. Tracy and H. Widom, Commun. Math. Phys. 177, 727 (1996).

[31] C. A. Tracy and H. Widom, Ann. of App. Proba. 17 (3), 953 (2007). 